\title{
Marcinkiewicz-Zygmund Inequalities for Polynomials in Bergman and Hardy Spaces
}

\author{
Karlheinz Gröchenig ${ }^{1}$ (D) Joaquim Ortega-Cerdà ${ }^{2}$
}

Received: 19 June 2020 / Accepted: 18 December 2020 / Published online: 3 February 2021

(c) The Author(s) 2021

\begin{abstract}
We study the relationship between sampling sequences in infinite dimensional Hilbert spaces of analytic functions and Marcinkiewicz-Zygmund inequalities in subspaces of polynomials. We focus on the study of the Hardy space and the Bergman space in one variable because they provide two settings with a strikingly different behavior.
\end{abstract}

Keywords Marcinkiewicz-Zygmund inequalities · Bergman space · Hardy space · Reproducing kernel

Mathematics Subject Classification 30E05 $\cdot 30 \mathrm{H} 20 \cdot 41 \mathrm{~A} 10 \cdot 42 \mathrm{~B} 30$

\section{Introduction}

Marcinkiewicz-Zygmund inequalities are finite-dimensional models for sampling in an infinite dimensional Hilbert or Banach space of functions. Originally they were studied in the context of interpolation by trigonometric polynomials. They became prominent in approximation theory, where they appear in quadrature rules and least square problems, and were usually studied in the context of orthogonal polynomials.

In an abstract setting one is given a reproducing kernel Hilbert space $\mathcal{H}$ on a set $S$ with reproducing kernel $k$ and a sequence of finite-dimensional subspaces $V_{n}$ such that

Karlheinz Gröchenig was supported in part by the project P31887-N32 of the Austrian Science Fund (FWF). J.O.C. has been partially supported by the Generalitat de Catalunya (Grant 2017 SGR 358) and the Spanish Ministerio de Ciencia, Innovación y Universidades (Project MTM2017-83499-P).

$凶 \quad$ Karlheinz Gröchenig

karlheinz.groechenig@univie.ac.at

Joaquim Ortega-Cerdà

jortega@ub.edu

1 Faculty of Mathematics, University of Vienna, Oskar-Morgenstern-Platz 1, 1090 Vienna, Austria

2 Departament de Matemàtiques i Informàtica, Universitat de Barcelona, Gran Via de les Corts Catalanes, 585, 08007 Barcelona, Spain 
$V_{n} \subseteq V_{n+1}$ and $\bigcup_{n} V_{n}$ is dense in $\mathcal{H}$. Each $V_{n}$ comes with its own reproducing kernel $k_{n}$, which is the orthogonal projection of $k$. A family of (finite) subsets $\Lambda_{n} \subseteq S$ is called a Marcinkiewicz-Zygmund family for $V_{n}$ in $\mathcal{H}$, if there exist constants $A, B>0$, the sampling constants, such that for all $n$ large, $n \geq n_{0}$,

$$
A\|p\|_{\mathcal{H}}^{2} \leq \sum_{\lambda \in \Lambda_{n}} \frac{|p(\lambda)|^{2}}{k_{n}(\lambda, \lambda)} \leq B\|p\|_{\mathcal{H}}^{2} \quad \text { for all } p \in V_{n}
$$

Thus a Marcinkiewicz-Zygmund family comes with a sequence of Marcinkiewicz-Zygmund inequalities, which are sampling inequalities for the finite-dimensional subspaces $V_{n}$. The point of the definition is that the sampling constants are independent of the subspace $V_{n}$.

The diagonal of the reproducing kernel $k_{n}$ furnishes the most natural choice of weights and goes back to the corresponding notion of interpolating sequences in reproducing kernel Hilbert spaces studied by Shapiro and Shields [26]. The weights are intrinsic to the underlying spaces $V_{n}$. Another hint for using $k_{n}$ comes from frame theory: Since $p(z)=\left\langle p, k_{n}(\cdot, z)\right\rangle$ for $p \in V_{n}$, the sampling inequality amounts to verifying that the normalized reproducing kernels $k_{n}(\cdot, \lambda) / k_{n}(\lambda, \lambda)^{1 / 2}$ form a frame in $V_{n}$ all whose elements have unit norm in $\mathcal{H}$.

Marcinkiewicz-Zygmund inequalities have been studied in many different contexts, e.g., for trigonometric polynomials $[6,22]$, for spaces of algebraic polynomials with respect to some measure $[14,15]$, for spaces of spherical harmonics on the sphere $[16,17,19]$, for spaces of eigenfunctions of the Laplacian on a compact Riemannian manifold [21], or even more generally for diffusion polynomials on a metric measure space [7]. In sampling theory Marcinkiewicz-Zygmund inequalities can be used to derive sampling theorems for bandlimited functions $[8,9]$.

In this paper we initiate the investigation of Marcinkiewicz-Zygmund inequalities for polynomials in spaces of analytic functions. As the reproducing kernel Hilbert space we take either the Bergman space $A^{2}(\mathbb{D})$ or the Hardy space $H^{2}(\mathbb{D})$ of analytic functions on the unit disk $\mathbb{D}$. The natural finite-dimensional subspaces will be the family of the polynomials $\mathcal{P}_{n}$ of degree $n$. In this context it is clear that an arbitrary set of at least $n+1$ distinct points yields a sampling inequality $A\|p\|^{2} \leq \sum_{\lambda \in \Lambda}|p(\lambda)|^{2} k_{n}(\lambda, \lambda)^{-1} \leq B\|p\|_{\mathcal{H}}^{2}$ for all $p \in \mathcal{P}_{n}$. The objective of Marcinkiewicz-Zygmund inequalities is to construct a sequence of finite sets $\Lambda_{n}$, such that the constants $A, B$ are independent of the degree $n$. The game is therefore to diligently keep track of the constants and show that they do not depend on the degree.

We will see that this problem is deeply related to sampling theorems for the full space $A^{2}$ or $H^{2}$. We say that $\Lambda \subseteq S$ is a sampling set for $\mathcal{H}$, if there exist constants $A, B>0$, such that

$$
A\|f\|_{\mathcal{H}}^{2} \leq \sum_{\lambda \in \Lambda} \frac{|f(\lambda)|^{2}}{k(\lambda, \lambda)} \leq B\|f\|_{\mathcal{H}}^{2} \quad \text { for all } f \in \mathcal{H}
$$

where now $k(z, w)$ is the reproducing kernel of $\mathcal{H}$. In our case $\mathcal{H}=A^{2}(\mathbb{D})$ or $=$ $H^{2}(\mathbb{D})$. 
Both the Bergman space and the Hardy space are reproducing kernel Hilbert spaces, in which the polynomials are dense and $k_{n}(\lambda, \lambda) \rightarrow k(\lambda, \lambda)$ pointwise. However, the underlying measures are different, and as a consequence the reproducing kernels and the implicit metrics are different. We will see that these differences imply a drastically different behavior of Marcinkiewicz-Zygmund families. In the Bergman space the points of a Marcinkiewicz-Zygmund family will be "uniformly" distributed in the entire disk, in Hardy space the points will cluster near the boundary of the disk. The concentration will depend on the degree. It will therefore be practical to introduce a notation of the relevant disks and annuli. For a fixed parameter $\gamma>0$, we will write

$$
B_{1-\gamma / n}=\left\{z \in \mathbb{D}:|z|<1-\frac{\gamma}{n}\right\}=B\left(0,1-\frac{\gamma}{n}\right)
$$

for the centered disk of radius $1-\frac{\gamma}{n}$, and

$$
C_{\gamma / n}=\left\{z \in \mathbb{D}: 1-\frac{\gamma}{n} \leq|z|<1\right\} \text {. }
$$

for the annulus of width $\gamma / n$ at the boundary of $\mathbb{D}$.

Our main result for the Bergman space $A^{2}(\mathbb{D})$ with norm $\|f\|_{A^{2}}^{2}=\frac{1}{\pi} \int_{\mathbb{D}}|f(z)|^{2} \mathrm{~d} z$, where $\mathrm{d} z$ is the area measure on $\mathbb{D}$, establishes a clear correspondence between sampling sets for $A^{2}(\mathbb{D})$ and Marcinkiewicz-Zygmund families for the polynomials $\mathcal{P}_{n}$ in $A^{2}$ as follows.

Theorem 1.1 (i) Assume that $\Lambda \subseteq \mathbb{D}$ is a sampling set for $A^{2}(\mathbb{D})$. Then for $\gamma>0$ small enough, the sets $\Lambda_{n}=\Lambda \cap B_{1-\gamma / n}$ form a Marcinkiewicz-Zygmund family for $\mathcal{P}_{n}$ in $A^{2}(\mathbb{D})$.

(ii) Conversely, if $\left(\Lambda_{n}\right)$ is a Marcinkiewicz-Zygmund family for the polynomials $\mathcal{P}_{n}$ in $A^{2}(\mathbb{D})$, then every weak limit of $\left(\Lambda_{n}\right)$ is a sampling set for $A^{2}(\mathbb{D})$.

See Sect. 2.5 for the definition of a weak limit of sets. The theorem shows that the construction of Marcinkiewicz-Zygmund families for the Bergman space is on the same level of difficulty as the construction of sampling sets for $A^{2}$. Fortunately, these sampling sets have been characterized completely in the deep work of Seip [23,24]. Sampling sets are completely determined by a suitable density, the Seip-Korenblum density. As a consequence of our main theorem, one can now give many examples of Marcinkiewicz-Zygmund families for $A^{2}$.

By contrast, the Hardy space does not admit any sampling sequences. By a theorem of Thomas [27] (Props. 2 and 3), a function $f \in H^{2}(\mathbb{D})$ satisfying $A\|f\|_{H^{2}}^{2} \leq$ $\sum_{\lambda \in \Lambda}|f(\lambda)|^{2} k(\lambda, \lambda)^{-1} \leq B\|f\|_{H^{2}}^{2}$ must be identical zero. Therefore there can be no analogue of Theorem 1.1(ii).

Despite the lack of a sampling theorem for $H^{2}(\mathbb{D})$ we can show the existence of Marcinkiewicz-Zygmund families for polynomials with a different method. The idea is to connect polynomials on the disk to polynomials on the torus in $L^{2}(\mathbb{T})$. By moving a Marcinkiewicz-Zygmund family for polynomials on $\mathbb{T}$ into the interior of $\mathbb{D}$, we obtain a Marcinkiewicz-Zygmund family for polynomials in Hardy space. Since the 
problem on the torus is well understood [22], we can derive a general construction of Marcinkiewicz-Zygmund families in $H^{2}(\mathbb{D})$.

Theorem 1.2 Assume that the family $\left(\widetilde{\Lambda_{n}}\right)=\left(\left\{e^{i v_{n, k}}: k=1, \ldots, L_{n}\right\}\right) \subseteq \mathbb{T}$ is $a$ Marcinkiewicz-Zygmund family for $\mathcal{P}_{n}$ on the torus, i.e.,

$$
A\|p\|_{L^{2}(\mathbb{T})}^{2} \leq \sum_{k=1}^{L_{n}} \frac{\left|p\left(e^{i v_{n, k}}\right)\right|^{2}}{n} \leq B\|p\|_{L^{2}(\mathbb{T})}^{2}
$$

for all polynomials $p$ of degree $n$.

Fix $\gamma>0$ arbitrary, choose $\rho_{n, k} \in\left[1-\frac{\gamma}{n}, 1\right)$ arbitrary, and set $\Lambda_{n}=\left\{\rho_{n, k} e^{i v_{n, k}}\right.$ : $\left.k=1, \ldots, L_{n}\right\} \subseteq C_{\gamma / n}$ for $n \in \mathbb{N}$. Then $\left(\Lambda_{n}\right)$ is a Marcinkiewicz-Zygmund family for $\mathcal{P}_{n}$ in $H^{2}(\mathbb{T})$.

This result provides a systematic construction of examples of Marcinkiewicz-Zygmund families for Hardy space, since Marcinkiewicz-Zygmund families for polynomials on the torus can be characterized almost completely by their density [22]. Marcinkiewicz-Zygmund families on the torus and more generally of orthogonal polynomials have been studied intensely in approximation theory, see $[6,8,14,15,18,20]$ for a sample of papers.

The technical heart of the matter is, as so often in complex analysis, the investigation and estimate of the reproducing kernels, namely the kernel $k(z, w)$ for the entire space $A^{2}$ or $H^{2}$ and the kernels $k_{n}(z, w)$ for the polynomials of degree $n$. The guiding principle is to sample the polynomials in the region where the diagonals $k(z, z)$ and $k_{n}(z, z)$ are comparable in size. One may call this region the "bulk" region. For the Bergman space the bulk region is the centered disk $B_{1-\gamma / n}$, because this is where the mass of polynomials of degree $n$ is concentrated. For the Hardy space the bulk region is the annulus $C_{\gamma / n}$, as the $H^{2}$-norm sees only the boundary behavior of functions in $H^{2}$.

Our main insight may be relevant in other settings. For instance, our main theorems can be extended to polynomials in weighted Bergman spaces or in Fock space [10]. We expect a version of Theorem 1.1 to hold for Bergman space on the unit ball in $\mathbb{C}^{n}$, though this will be more technical to elaborate.

The paper is organized as follows: In Sect. 2 we treat the theory of MarcinkiewiczZygmund families in the Bergman space $A^{2}(\mathbb{D})$ and in Sect. 3 we treat the Hardy space $H^{2}(\mathbb{D})$. Each section starts with the necessary background, the comparisons of the various reproducing kernels and the main contribution to the norms. Then we formulate and prove the main results about Marcinkiewicz-Zygmund families.

Throughout we will use the notation $\lesssim$ to abbreviate an inequality $f \leq C g$ where the constants is independent of the essential input, which in our case will be the degree of the polynomial. To indicate the dependence of the constant on some parameter $\gamma$, say, we will write $\lesssim \gamma$. As usual, $f \asymp g$ means that both $f \lesssim g$ and $g \lesssim f$ hold. 


\section{Bergman Space}

\subsection{Basic Facts}

The Bergman space $A^{2}=A^{2}(\mathbb{D})$ consists of all analytic functions on the unit disk $\mathbb{D}$ with finite norm

$$
\|f\|_{A^{2}}=\left(\frac{1}{\pi} \int_{\mathbb{D}}|f(z)|^{2} \mathrm{~d} z\right)^{1 / 2}
$$

where $\mathrm{d} z$ is the area measure on $\mathbb{D}$. For a detailed exposition of Bergman spaces we refer to the excellent monographs $[4,13]$, both of which contain an entire chapter on sampling in Bergman space.

The monomials $z \mapsto z^{k}$ are orthogonal with norm $\left\|z^{k}\right\|_{A^{2}}^{2}=\frac{1}{k+1}$. Consequently the norm of $f(z)=\sum_{k=0}^{\infty} a_{k} z^{k}$ is

$$
\|f\|_{A^{2}}^{2}=\sum_{k=0}^{\infty}\left|a_{k}\right|^{2} \frac{1}{k+1}
$$

Let $p(z)=\sum_{k=0}^{n} a_{k} z^{k} \in \mathcal{P}_{n}$, then its norm on a disk $B_{\rho}, \rho<1$, is given by

$$
\begin{aligned}
\frac{1}{\pi} \int_{B_{\rho}}|p(z)|^{2} \mathrm{~d} z & =\frac{1}{\pi} \sum_{k, l=0}^{n} a_{k} \overline{a_{l}} \int_{B_{\rho}} z^{k} \bar{z}^{l} \mathrm{~d} z \\
& =\frac{1}{\pi} 2 \pi \sum_{k=0}^{n}\left|a_{k}\right|^{2} \int_{0}^{\rho} r^{2 k} r \mathrm{~d} r \\
& =\sum_{k=0}^{n}\left|a_{k}\right|^{2} \rho^{2 k+2} \frac{1}{k+1} .
\end{aligned}
$$

For $p \in \mathcal{P}_{n}$, we therefore have

$$
\frac{1}{\pi} \int_{B_{\rho}}|p(z)|^{2} \mathrm{~d} z \geq \rho^{2 n+2}\|p\|_{A^{2}}^{2}
$$

To obtain a bound independent of $n$, we need to choose $\rho_{n}$ such that $\rho_{n}^{2 n} \geq A$ for all $n$. By picking $\rho_{n}=1-\frac{\gamma}{n}$, we find

$$
e^{-2 \gamma} \leq\left(1-\frac{\gamma}{n}\right)^{n} \leq e^{-\gamma} \quad \forall n>2 \gamma .
$$

In the following we will use these inequalities abundantly.

Corollary 2.1 If $p \in \mathcal{P}_{n}$, then for every $\gamma>0$ and $n>2 \gamma$

$$
\frac{1}{\pi} \int_{C_{\gamma / n}}|p(w)|^{2} \mathrm{~d} w \leq\|p\|_{A^{2}}^{2}\left(1-\left(1-\frac{\gamma}{n}\right)^{2 n+2}\right) \leq\|p\|_{A^{2}}^{2}\left(1-\frac{1}{4} e^{-4 \gamma}\right) .
$$


Furthermore, for $\epsilon>0$ there exists $\gamma>0$ (small enough) such that

$$
\frac{1}{\pi} \int_{C_{\gamma / n}}|p(w)|^{2} \mathrm{~d} w \leq \epsilon\|p\|_{A^{2}}^{2} \quad \text { for all } p \in \mathcal{P}_{n}
$$

independent of $n$.

Proof With (7) and the partition $\mathbb{D}=B_{1-\gamma / n} \cup C_{\gamma / n}$ we obtain

$$
\begin{aligned}
\frac{1}{\pi} \int_{C_{\gamma / n}}|p(w)|^{2} \mathrm{~d} w & =\|p\|_{A^{2}}^{2}-\frac{1}{\pi} \int_{B_{1-\gamma / n}}|p(w)|^{2} \mathrm{~d} w \\
& \leq\|p\|_{A^{2}}^{2}\left(1-\left(1-\frac{\gamma}{n}\right)^{2 n+2}\right) \leq\|p\|_{A^{2}}^{2}\left(1-\frac{1}{4} e^{-4 \gamma}\right),
\end{aligned}
$$

since $\left(1-\frac{\gamma}{n}\right)^{2 n+2} \geq e^{-4 \gamma}\left(1-\frac{\gamma}{n}\right)^{2} \geq e^{-4 \gamma} / 4$ for $n \geq 2 \gamma$. Also as $\gamma \rightarrow 0$, $\inf _{n \in \mathbb{N}}\left(1-\frac{\gamma}{n}\right)^{2 n+2} \rightarrow 1$.

\subsection{The Bergman Kernels}

Since $\sqrt{k+1} z^{k}$ is an orthonormal basis for $\mathcal{P}_{n}$ in $A^{2}(\mathbb{D})$, the reproducing kernel of $\mathcal{P}_{n}$ in $A^{2}$ is given by

$$
k_{n}(z, w)=\sum_{k=0}^{n}(k+1)(z \bar{w})^{k}=\frac{1+(n+1)(z \bar{w})^{n+2}-(n+2)(z \bar{w})^{n+1}}{(1-z \bar{w})^{2}} .
$$

As $n \rightarrow \infty$, the kernel tends to the Bergman kernel of $A^{2}$,

$$
k(z, w)=\frac{1}{(1-z \bar{w})^{2}} \quad z, w \in \mathbb{D} .
$$

We first compare these kernels in two regimes, namely the "bulk" regions $B_{1-\gamma / n}$ and the boundary region $C_{\gamma / n}$.

Lemma 2.2 Let $k_{n}(z, w)$ be the reproducing kernel of $\mathcal{P}_{n}$ in $A^{2}$ and $\gamma>0$ be arbitrary.

(i) If $|z| \leq 1-\frac{\gamma}{n}$, then $k_{n}(z, z) \asymp k(z, z)$ for $n$ large enough, $n \geq n_{\gamma}$, precisely

$$
c_{\gamma} k(z, z) \leq k_{n}(z, z) \leq k(z, z)=\frac{1}{\left(1-|z|^{2}\right)^{2}},
$$

where $c_{\gamma}>0$ can be chosen as $c_{\gamma}=1-e^{-2 \gamma}(1+2 \gamma)$.

(ii) If $1-\frac{\gamma}{n} \leq|z|<1$ and $n>\max (\gamma, 3)$, then $k_{n}(z, z) \asymp n^{2}$, precisely,

$$
\frac{e^{-4 \gamma}}{4} n^{2} \leq k_{n}(z, z) \leq n^{2}
$$


Thus inside the disk $B_{1-\gamma / n}$ the reproducing kernel for $\mathcal{P}_{n}$ behaves like the reproducing kernel of the full space $A^{2}$, whereas in the annulus it behaves like $n^{2}$ and is much smaller than $k(z, z)$ near the boundary of $\mathbb{D}$.

Proof (i) It is always true that $k_{n}(z, z) \leq k(z, z)=\frac{1}{\left(1-|z|^{2}\right)^{2}}$. For the lower bound, let $r=|z|$ and observe that

$$
k_{n}(z, z)=k(z, z)\left(1-(n+2) r^{2 n+2}+(n+1) r^{2 n+4}\right)=k(z, z)(1-q(r)) .
$$

Since $q(r)=(n+2) r^{2 n+2}-(n+1) r^{2 n+4}$ is increasing on $(0,1)$ and $r \leq 1-\frac{\gamma}{n}$, we need an upper estimate for $q$ at $1-\frac{\gamma}{n}$. With the help of (8) and some algebra we write $q$ as

$$
\begin{aligned}
q\left(1-\frac{\gamma}{n}\right) & =\left(1-\frac{\gamma}{n}\right)^{2 n+2}\left(n+2-(n+1)\left(1-\frac{\gamma}{n}\right)^{2}\right) \\
& =\left(1-\frac{\gamma}{n}\right)^{2 n}\left(1-\frac{\gamma}{n}\right)^{2}\left(1+2 \gamma+\frac{\gamma}{n}(2-\gamma)-\frac{\gamma^{2}}{n^{2}}\right) \\
& =\left(1-\frac{\gamma}{n}\right)^{2 n}\left(1+2 \gamma-\frac{5 \gamma^{2}}{n}+\frac{\alpha_{\gamma}}{n^{2}}+\frac{\beta_{\gamma}}{n^{3}}\right)
\end{aligned}
$$

for some constants $\alpha_{\gamma}, \beta_{\gamma}$. Using (8) and a sufficiently large $n, n \geq n_{\gamma}$ say, the final estimate for $q$ is

$$
q\left(1-\frac{\gamma}{n}\right) \leq e^{-2 \gamma}(1+2 \gamma)<1, \text { for } n \geq n_{\gamma}
$$

Combined with (13) we have the lower estimate

$$
k_{n}(z, z) \geq\left(1-e^{-2 \gamma}(1+2 \gamma)\right) k(z, z)=c_{\gamma} k(z, z)
$$

for $|z| \leq 1-\frac{\gamma}{n}$ and $n \geq n_{\gamma}$.

(ii) If $1-\frac{\gamma^{n}}{n} \leq|z|<1$, then

$$
k_{n}(z, z)=\sum_{k=0}^{n}|z|^{2 k}(k+1) \leq \sum_{k=0}^{n}(k+1)=\frac{(n+1)(n+2)}{2} \leq n^{2},
$$

for $n \geq 4$, and, for $n \geq 2 \gamma$,

$$
k_{n}(z, z)=\sum_{k=0}^{n}|z|^{2 k}(k+1) \geq \sum_{k \geq n / 2}^{n}\left(1-\frac{\gamma}{n}\right)^{2 n} \frac{n}{2} \geq e^{-4 \gamma} \frac{n^{2}}{4} .
$$

We will also need some information about the behavior of the reproducing kernels $k_{n}$ near the diagonal. It will be convenient to use the normalized reproducing kernel $\kappa_{n}(z, w)$ for $\mathcal{P}_{n}$ at the point $w$, i.e., $\kappa_{n}(z, w)=k_{n}(z, w) / \sqrt{k_{n}(w, w)}$. It satisfies $\left\|\kappa_{n}(\cdot, w)\right\|_{A^{2}}=1$, and as we have observed in (11), if $w \in B_{1-\gamma / n}$, then $\kappa_{n}(w, w) \asymp$ 
$\frac{1}{1-|w|^{2}}$. The following lemma collects the properties of $\kappa_{n}$ near the diagonal in the different regimes in $\mathbb{D}$.

Lemma 2.3 (i) There is a constant $\gamma_{0}$ such that for all $w \in B_{1-\gamma_{0} / n}$ and $z \in$ $B\left(w, 0.5\left(1-|w|^{2}\right)\right)$ we have

$$
\frac{1}{4\left(1-|w|^{2}\right)} \leq\left|\kappa_{n}(z, w)\right| \leq \frac{9}{4\left(1-|w|^{2}\right)}
$$

(ii) For every $\gamma>0$ there are $K>0$ and $\epsilon>0$ depending only on $\gamma$ such that

$$
\frac{n}{K} \leq\left|\kappa_{n}(z, w)\right| \leq K n \quad \text { for all } w \in C_{\gamma / n}, z \in B(w, \epsilon / n)
$$

and $n>\gamma$.

Proof (i) If $z \in B\left(w, 0.5\left(1-|w|^{2}\right)\right)$, then

$$
|1-z \bar{w}|=\left.|1-| w\right|^{2}-\bar{w}(z-w)\left|\geq\left(1-|w|^{2}\right)-\right| \bar{w}(z-w) \mid \geq \frac{1}{2}\left(1-|w|^{2}\right),
$$

and likewise $|1-z \bar{w}| \leq \frac{3}{2}\left(1-|w|^{2}\right)$. To obtain similar bounds for the numerator, it suffices to prove that

$$
\left|(n+2)(z \bar{w})^{n+1}-(n+1)(z \bar{w})^{n+2}\right|<\frac{1}{2} .
$$

for $\gamma$ sufficiently large. We replace $n+1$ by $n$ and rewrite this expression as

$$
\begin{aligned}
\left|(n+1)(z \bar{w})^{n}-n(z \bar{w})^{n+1}\right| & =\left|n(z \bar{w})^{n}\left(\frac{n+1}{n}-z \bar{w}\right)\right| \\
& \leq n|w|^{n}\left(|1-z \bar{w}|+\frac{1}{n}\right) \\
& \leq n|w|^{n} \frac{3}{2}\left(1-|w|^{2}\right)+|w|^{n}
\end{aligned}
$$

The map $r \rightarrow r^{n}\left(1-r^{2}\right)$ in (15) is increasing on $\left[0,\left(\frac{n}{n+2}\right)^{1 / 2}\right]$. Therefore its maximum is taken at $r=1-\frac{\gamma}{n}$, provided that $\gamma \geq 1$ and $n \geq 1$. So we obtain the estimate $\left|(n+1)(z \bar{w})^{n}-n(z \bar{w})^{n+1}\right| \leq 3 n\left(1-\frac{\gamma}{n}\right)^{n} \frac{\gamma}{n}+\left(1-\frac{\gamma}{n}\right)^{n} \leq 3 e^{-\gamma} \gamma+e^{-\gamma}<1 / 2$ for $\gamma$ large enough. In fact, we may take $\gamma \geq 3$.

(ii) We know from (12) that $k_{n}(w, w) \asymp n^{2}$. Furthermore

$$
\left|\kappa_{n}^{\prime}(z, w)\right|=\frac{1}{k_{n}(w, w)^{1 / 2}}\left|\frac{\partial k_{n}(z, w)}{\partial z}\right| \lesssim \frac{1}{n}\left|\sum_{k=1}^{n} k(k+1)(z \bar{w})^{k-1} \bar{w}\right| \leq C n^{2}
$$

Thus, if $z \in B(w, \epsilon / n)$, then $\kappa_{n}(z) \geq \frac{e^{-2 \gamma}}{2} n-C \epsilon n$. Now take $\epsilon \leq \frac{C e^{-2 \gamma}}{4}$ and $K=\frac{e^{-2 \gamma}}{4}$ and (ii) follows. 


\subsection{Separation and Carleson-Type Conditions}

We first study the upper estimate of the Marcinkiewicz-Zygmund inequalities and derive a geometric description.

Let $d(z, w)=\left|\frac{z-w}{1-z \bar{w}}\right|$ be the pseudohyperbolic metric on $\mathbb{D}$. We denote $\Delta(w, \rho)=$ $\{z \in \mathbb{D}: d(z, w)<\rho\}$ the hyperbolic disk in $\mathbb{D}$. While $\Delta(w, \rho)$ is also a Euclidean disk (albeit with a different center and radius), it will be more convenient for us to compare it to a Euclidean disk with the same center $w$. In fact, we have the following inclusions:

$$
B\left(w, \frac{\rho}{1+\rho}\left(1-|w|^{2}\right)\right) \subseteq \Delta(w, \rho) \subseteq B\left(w, \frac{\rho}{1-\rho}\left(1-|w|^{2}\right)\right),
$$

where the latter inclusion holds for $\rho<1 / 2$.

A set $\Lambda \subseteq \mathbb{D}$ is called uniformly discrete, if there is a $\delta^{\prime}>0$ such that $d(\lambda, \mu) \geq \delta^{\prime}$ for all $\lambda, \mu \in \Lambda, \lambda \neq \mu$. In view of (16) this is equivalent to the fact that the Euclidean balls $B(\lambda, \delta(1-|\lambda|)), \lambda \in \Lambda$ are disjoint in $\mathbb{D}$ for some $\delta>0$. We refer to this condition as $\Lambda$ being $\delta$-separated.

In $A^{2}(\mathbb{D})$ the upper estimate of the sampling inequality is characterized by the following geometric condition. See [4, Sect. 2.11, Thm. 15] and [24].

Proposition 2.4 For $\Lambda \subseteq \mathbb{D}$ the following conditions are equivalent:

(i) The inequality $\sum_{\lambda \in \Lambda} \frac{|f(\lambda)|^{2}}{k(\lambda, \lambda)} \leq B\|f\|_{A^{2}}^{2}$ holds for all $f \in A^{2}(\mathbb{D})$.

(ii) $\Lambda$ is a finite union of uniformly discrete sets.

(iii) $\sup _{w \in \mathbb{D}} \#(\Lambda \cap \Delta(w, \rho))<\infty$ for some (hence all) $\rho \in(0,1)$.

Condition (i) is often formulated by saying that the measure $\sum_{\lambda \in \Lambda} k(\lambda, \lambda) \delta_{\lambda}$ is a Carleson measure for $A^{2}$.

If we add the (much more difficult) lower sampling inequality to the assumptions, we also have the following lemma of Seip [24] (see Lemma 5.2 and Thm. 7.1)

Lemma 2.5 If $\Lambda$ is a sampling set for $A^{2}(\mathbb{D})$, then $\Lambda$ contains a uniformly discrete set $\Lambda^{\prime} \subseteq \Lambda$ that is also sampling for $A^{2}(\mathbb{D})$.

The proof of the implication $(i i) \Rightarrow$ (i) yields a local version of the Bessel inequality that will be needed.

Lemma 2.6 Let $\Lambda$ be a $\delta$-separated set and let $\gamma>0$. Then there exists a constant $C=C(\delta)$ and $\gamma^{\prime}>\gamma$, such that

$$
\sum_{\lambda \in \Lambda \cap C_{\gamma / n}} \frac{|f(\lambda)|^{2}}{k(\lambda, \lambda)} \leq C \int_{C_{\gamma^{\prime} / n}}|f(w)|^{2} \mathrm{~d} w \quad \text { for all } f \in A^{2}
$$

The constants depend only on the separation via $\gamma^{\prime}=(1+\delta) \gamma$ and $C=\frac{4}{\pi \delta^{2}}$.

Proof See [4], Sect. 2.11, Lemma 14. For completeness we include the proof. 
By assumption the Euclidean balls $B_{\lambda}=B(\lambda, \delta(1-|\lambda|)) \subseteq \mathbb{D}$ for $\lambda \in C_{\gamma / n}$ are disjoint. Since $\left|B_{\lambda}\right| k(\lambda, \lambda)=\pi \delta^{2}(1-|\lambda|)^{2}\left(1-|\lambda|^{2}\right)^{-2} \geq \pi \delta^{2} / 4$, the submean-value property for $|f|^{2}$ yields the estimate

$$
\frac{|f(z)|^{2}}{k(\lambda, \lambda)} \leq \frac{1}{k(\lambda, \lambda)\left|B_{\lambda}\right|} \int_{B_{\lambda}}|f(w)|^{2} \mathrm{~d} w \leq \frac{4}{\pi \delta^{2}} \int_{B_{\lambda}}|f(w)|^{2} \mathrm{~d} w .
$$

By summing over $\lambda \in \Lambda \cap C_{\gamma / n}$ and using the disjointness of the disks $B_{\lambda}$ we obtain that

$$
\sum_{\lambda \in C_{\gamma / n}} \frac{|f(\lambda)|^{2}}{k(\lambda, \lambda)} \leq \frac{4}{\pi \delta^{2}} \sum_{\lambda \in C_{\gamma / n}} \int_{B_{\lambda}}|f(w)|^{2} \mathrm{~d} w=\frac{4}{\pi \delta^{2}} \int_{\bigcup_{\lambda \in C_{\gamma / n}} B_{\lambda}}|f(w)|^{2} \mathrm{~d} w .
$$

Since dist $\left(0, B_{\lambda}\right)=|\lambda|-\delta(1-|\lambda|)=|\lambda|(1+\delta)-\delta \geq\left(1-\frac{\gamma}{n}\right)(1+\delta)-\delta=$ $1-\frac{(1+\delta) \gamma}{n}=1-\frac{\gamma^{\prime}}{n}$, the disks $B_{\lambda}$ are contained in the annulus $C_{\gamma^{\prime} / n}$. Since they are disjoint, we obtain

$$
\int_{\bigcup_{\lambda \in C_{\gamma / n}} B_{\lambda}}|f(w)|^{2} \mathrm{~d} w \leq \int_{C_{\gamma^{\prime} / n}}|f(w)|^{2} \mathrm{~d} w
$$

If $\Lambda$ is a union of $K$ separated sets $\Lambda_{m}$, we apply the above argument to each $\Lambda_{m}$ and then obtain the constant $C=\frac{4 K}{\pi \delta^{2}}$.

Next we develop a geometric description for the upper Marcinkiewicz-Zygmund inequalities in $A^{2}$ that is similar to Proposition 2.4. For this we estimate the number of points of a Marcinkiewicz-Zygmund family in the relevant regions of the disk, namely in the bulk $B_{1-\gamma / n}$, the annulus $C_{\gamma / n}$, and in the cells $B(w, \epsilon / n) \cap \mathbb{D}$ for $w$ near the boundary of $\mathbb{D}$.

Proposition 2.7 Assume that $\left(\Lambda_{n}\right)$ satisfies the upper Marcinkiewicz-Zygmund inequalities (1) for $\mathcal{P}_{n}$ in $A^{2}(\mathbb{D})$.

(i) Then for every $\gamma>0$

$$
\#\left(\Lambda_{n} \cap C_{\gamma / n}\right) \leq C n
$$

(ii) There are $\gamma_{0}>0\left(\gamma_{0} \approx 3\right)$ and $C>0$ such that

$$
\#\left(\Lambda_{n} \cap B(z, 0.5(1-|z|))\right) \leq C, \quad \forall z \in B_{1-\gamma_{0} / n}
$$

As a consequence, $\Lambda_{n} \cap B_{1-\gamma_{0} / n}$ is a disjoint union of at most $C$ uniformly discrete subsets with a separation $\delta$ independent of $n$.

(iii) For every $\gamma>0$ there is $\epsilon>0$ such that

$$
\#\left(\Lambda_{n} \cap B\left(z, \frac{\epsilon}{n}\right)\right) \leq C, \quad \forall z \in C_{\gamma / n} .
$$

The constants depend only on $\gamma$ and the upper Marcinkiewicz-Zygmund bound B. 
Proof In each case we test the upper Marcinkiewicz-Zygmund inequalities against a suitable polynomial in $\mathcal{P}_{n}$.

(i) Choose the monomial $p(z)=z^{n} \in \mathcal{P}_{n}$ with norm $\|p\|_{A^{2}}^{2}=\frac{1}{n+1}$. Since for $\lambda \in C_{\gamma / n},|p(\lambda)| \asymp_{\gamma} 1$ by (8) and $k_{n}(\lambda, \lambda) \asymp_{\gamma} n^{2}$ by (12), we obtain

$$
\frac{1}{n^{2}} \#\left(\Lambda_{n} \cap C_{\gamma / n}\right) \asymp_{\gamma} \sum_{\lambda \in \Lambda_{n} \cap C_{\gamma / n}} \frac{|p(\lambda)|^{2}}{k_{n}(\lambda, \lambda)} \leq B\|p\|_{A^{2}}^{2}=\frac{B}{n+1} .
$$

This implies \# $\left(\Lambda_{n} \cap C_{\gamma / n}\right) \lesssim n$.

(ii) We choose $\kappa_{n}(\cdot, z) \in \mathcal{P}_{n}$. For $z \in B_{1-\gamma / n}$ and $\lambda \in B(z, 0.5(1-|z|))$ we have $\left|\kappa_{n}(\lambda, z)\right|^{2} \asymp\left(1-|z|^{2}\right)^{-2}$ by Lemma 2.3(i), and $k_{n}(\lambda, \lambda) \asymp\left(1-|\lambda|^{2}\right)^{-2} \asymp$ $\left(1-|z|^{2}\right)^{-2}$ by Lemma 2.2. We conclude that $\frac{\left|\kappa_{n}(\lambda, z)\right|^{2}}{k_{n}(\lambda, \lambda)} \geq C$ for some constant, and thus

$$
C \#\left(\Lambda_{n} \cap B(z, 0.5(1-|z|))\right) \leq \sum_{\lambda \in B(z, 0.5(1-|z|))} \frac{\left|\kappa_{n}(\lambda, z)\right|^{2}}{k_{n}(\lambda, \lambda)} \leq B\left\|\kappa_{n}(\cdot, z)\right\|_{A^{2}}^{2}=B .
$$

The relative separation follows as in [4], Sect. 2.11, Lemma 16.

(iii) Again we choose $\kappa_{n}(\cdot, z)$, this time for $z \in C_{\gamma / n}$. By Lemma $2.3\left|\kappa_{n}(\lambda, z)\right|^{2} \asymp n^{2}$ for $\lambda \in B(z, \epsilon / n)$. Likewise $k_{n}(z, z) \asymp n^{2}$ for $z \in C_{\gamma / n}$ by Lemma 2.2. Thus

$$
C \#\left(\Lambda_{n} \cap B\left(z, \frac{\epsilon}{n}\right)\right) \leq \sum_{\lambda \in \Lambda_{n} \cap B\left(z, \frac{\epsilon}{n}\right)} \frac{\left|\kappa_{n}(\lambda, z)\right|^{2}}{k_{n}(\lambda, \lambda)} \leq B\left\|\kappa_{n}(\cdot, z)\right\|_{A^{2}}^{2}=B
$$

As a consequence we see that the cardinality of a Marcinkiewicz-Zygmund family for polynomials obeys the correct order of growth.

Corollary 2.8 If $\left(\Lambda_{n}\right)$ satisfies the upper Marcinkiewicz-Zygmund inequality, then $\# \Lambda_{n} \lesssim n$.

Proof Choose $\gamma$ large enough as in Lemma 2.7(ii). We cover $B_{1-\gamma / n}=\Delta\left(0,1-\frac{\gamma}{n}\right)$ with hyperbolic disks $\Delta\left(z_{j}, \frac{1}{3}\right) \subseteq B\left(z_{j}, 0.5\left(1-\left|z_{j}\right|\right)\right)$, such that the disks $\Delta\left(z_{j}, \frac{1}{6}\right)$ are disjoint. Since the hyperbolic area of $B_{1-\gamma / n}$ is $\int_{B_{1-\gamma / n}} \frac{\mathrm{d} z}{\left(1-|z|^{2}\right)^{2}} \leq n / \gamma$, we need at most $c n / \gamma$ disks (where $c^{-1}$ is the hyperbolic area of $\Delta\left(z_{j}, \frac{1}{6}\right)$ ). By Lemma 2.7(ii) every hyperbolic disk $B\left(z_{j}, 0.5\left(1-\left|z_{j}\right|\right)\right)$ contains $C$ points, so that $\#\left(\Lambda_{n} \cap B_{1-\gamma / n}\right) \leq$ $C n$ for a suitable constant.

By Lemma 2.7(i) \# $\left(\Lambda_{n} \cap C_{\gamma / n}\right) \lesssim n$ as well.

We can now formulate a geometric description of the upper Marcinkiewicz-Zygmund inequality (1), that is, the Bessel inequality of the normalized reproducing kernels.

Theorem 2.9 For a family $\left(\Lambda_{n}\right) \subseteq \mathbb{D}$ the following conditions are equivalent: 
(i) For all $n \geq n_{0}$

$$
\sum_{\lambda \in \Lambda_{n}} \frac{|p(\lambda)|^{2}}{k_{n}(\lambda, \lambda)} \leq B\|p\|_{A^{2}}^{2}, \quad \forall p \in \mathcal{P}_{n}
$$

(ii) There are $\gamma>0$ and $C>0$ such that $\left(\Lambda_{n}\right)$ satisfies

$$
\begin{aligned}
\#\left(\Lambda_{n} \cap B(w, 0.5(1-|w|))\right) \leq C & \forall w \in B_{1-\gamma / n} \\
\#\left(\Lambda_{n} \cap B(w, 1 / n)\right) \leq C & \forall w \in C_{\gamma / n} .
\end{aligned}
$$

Proof (i) $\Rightarrow$ (ii): This is Proposition 2.7.

(ii) $\Rightarrow$ (i): We write

$$
\sum_{\lambda \in \Lambda_{n}} \frac{|p(\lambda)|^{2}}{k_{n}(\lambda, \lambda)}=\sum_{\lambda \in \Lambda_{n} \cap B_{1-\gamma / n}} \cdots+\sum_{\lambda \in \Lambda_{n} \cap C_{\gamma / n}} \cdots
$$

and control each term with the appropriate geometric condition.

On $B_{1-\gamma / n}$ we may replace $k_{n}$ by $k$ (Lemma 2.2). Since $\Lambda_{n} \cap B_{1-\gamma / n}$ is a union of at most $C$ uniformly discrete sets with fixed separation independent of $n$ by our assumption (20), Proposition 2.4-or the appropriate version of Lemma 2.6-implies that

$$
\sum_{\lambda \in \Lambda_{n} \cap B_{1-\gamma / n}} \frac{|p(\lambda)|^{2}}{k_{n}(\lambda, \lambda)} \lesssim \gamma \sum_{\lambda \in \Lambda_{n} \cap B_{1-\gamma / n}} \frac{|p(\lambda)|^{2}}{k(\lambda, \lambda)} \lesssim\|p\|_{A^{2}}^{2}
$$

On $C_{\gamma / n}$ we have $k_{n}(\lambda, \lambda)|B(\lambda, 1 / n)| \asymp 1$ by (12); therefore using the submean-value property we obtain

$$
\begin{aligned}
\sum_{\lambda \in C_{\gamma / n}} \frac{|p(\lambda)|^{2}}{k_{n}(\lambda, \lambda)} & \leq \sum_{\lambda \in C_{\gamma / n}} \frac{1}{k_{n}(\lambda, \lambda)|B(\lambda, 1 / n)|} \int_{B(\lambda, 1 / n)}|p(z)|^{2} \mathrm{~d} z \\
& \lesssim \int\left(\sum_{\lambda \in \Lambda_{n} \cap C_{\gamma / n}} \chi_{B(\lambda, 1 / n)}(z)\right)|p(z)|^{2} \mathrm{~d} z .
\end{aligned}
$$

The integral is over the slightly larger annulus $C_{(\gamma+1) / n} \supseteq \bigcup_{\lambda \in C_{\gamma / n}} B(\lambda, 1 / n)$, and by assumption (21) the sum in the integral is bounded, whence $\sum_{\lambda \in C_{\gamma / n}} \frac{|p(\lambda)|^{2}}{k_{n}(\lambda, \lambda)} \lesssim$ $\|p\|_{A^{2}}^{2}$.

In a more general formulation we can use measures in condition (i) instead of discrete samples. This motivates the following definition.

Definition 2.1 We say that a sequence of measures $\left\{\mu_{n}\right\}$ defined on the unit disk form a Carleson sequence for polynomials in the Bergman space if there is a constant $C>0$ such that 


$$
\int_{\mathbb{D}}|p(z)|^{2} \mathrm{~d} \mu_{n}(z) \leq C \int_{\mathbb{D}}|p(z)|^{2} \mathrm{~d} z \quad \text { for all } p \in \mathcal{P}_{n}
$$

uniformly in $n$.

Thus a sequence $\left(\Lambda_{n}\right) \subseteq \mathbb{D}$ satisfies the upper inequality in (1) if and only if the measures $\mu_{n}=\sum_{\lambda \in \Lambda_{n}} \frac{\delta_{\lambda}}{k_{n}(\lambda, \lambda)}$ form a Carleson sequence.

Theorem 2.10 A sequence of measures $\mu_{n}$ is a Carleson sequence for polynomials in the Bergman space if and only if there exist $\gamma>0$ and $C>0$ such that

$$
\begin{aligned}
\mu_{n}\left(B\left(z, 0.5\left(1-|z|^{2}\right)\right)\right) & \leq C\left(1-|z|^{2}\right)^{2} \quad \forall z \in B_{1-\gamma / n}, \\
\mu_{n}(B(z, 1 / n)) & \leq C / n^{2} \quad \forall z \in C_{\gamma / n} .
\end{aligned}
$$

The proof is similar to the proof of Theorem 2.9. As we will not need the general statement about Carleson sequences, we omit the details.

Remark The geometric condition in Theorems 2.9 and 2.10 can be stated more concisely in terms of the first Bergman metric $\rho_{n}=k_{n}(z, z) \mathrm{d} s^{2}$, see [1, p. 32] instead of the Euclidean metric. This is not to be confused with the more usual second Bergman metric given by $\Delta \log k_{n}(z, z) \mathrm{d} s^{2}$. The reformulation is as follows: if $D_{n}(z, r)$ denotes a disk in the first Bergman metric $\rho_{n}$, then a sequence of measures $\left\{\mu_{n}\right\}$ is a Carleson sequence if and only if there are $r>0$ and $C>0$ such that $\mu_{n}\left(D_{n}(z, r)\right) \leq C\left|D_{n}(z, r)\right|$, for all $n>0$ and for all $z \in \mathbb{D}$, where $|D(z, r)|$ is the Lebesgue measure of $D(z, r)$. The conditions of (ii) in Theorem 2.9 are then equivalent to $\#\left(\Lambda_{n} \cap D_{n}(z, r)\right) \leq C$ for all $z \in \mathbb{D}$.

This reformulation is of course similar to the characterization of the Carleson measures for the Bergman space obtained in [12]. The geometric description is $\mu(B(z, 0.5(1-|z|))) \lesssim|B(z, 0.5(1-|z|))|$ for all $z \in \mathbb{D}$.

\subsection{Sampling Implies Marcinkiewicz-Zygmund Inequalities}

After these preparations we can show that every sampling set for $A^{2}(\mathbb{D})$ generates a Marcinkiewicz-Zygmund family for the polynomials.

Theorem 2.11 Assume that $\Lambda \subseteq \mathbb{D}$ is a sampling set for $A^{2}(\mathbb{D})$. Then for $\gamma>0$ small enough, the sets $\Lambda_{n}=\Lambda \cap B_{1-\gamma / n}$ form a Marcinkiewicz-Zygmund family for $\mathcal{P}_{n}$ in $A^{2}(\mathbb{D})$.

Proof The assumption means that there exist $A, B>0$, such that

$$
A\|f\|_{A^{2}}^{2} \leq \sum_{\lambda \in \Lambda} \frac{|f(\lambda)|^{2}}{k(\lambda, \lambda)} \leq B\|f\|_{A^{2}}^{2} \text { for all } f \in A^{2} .
$$


Since $\Lambda$ is sampling for $A^{2}$, by Lemma 2.5 it contains a uniformly discrete set $\Lambda^{\prime}$ with separation constant $\delta$, say. We now choose $\gamma>0$ so small that

$$
1-\left(1-\frac{(1+\delta) \gamma}{n}\right)^{2 n+2} \leq \frac{A \delta^{2}}{8} \quad \text { for all } n>2 \gamma
$$

This is possible because $\left(1-\frac{(1+\delta) \gamma}{n}\right)^{2 n+2} \geq e^{-4(1+\delta) \gamma}\left(1-\frac{(1+\delta) \gamma}{n}\right)^{2}$ by the lower estimate in (8), and thus tends to 1 uniformly in $n$, as $\gamma \rightarrow 0$. Note that the choice of $\gamma$ depends on the lower sampling constant $A$ and the separation of $\Lambda^{\prime}$.

We now apply Lemma 2.6 and Corollary 2.1 to the points $\Lambda \backslash \Lambda_{n}=\Lambda \cap C_{\gamma / n}$. Set $\gamma^{\prime}=(1+\delta) \gamma$. Then for $p \in \mathcal{P}_{n}$ we obtain

$$
\begin{aligned}
\sum_{\lambda \in \Lambda \backslash \Lambda_{n}} \frac{|p(\lambda)|^{2}}{k(\lambda, \lambda)} & \leq \frac{4}{\pi \delta^{2}} \int_{C_{\gamma^{\prime} / n}}|p(w)|^{2} \mathrm{~d} w \\
& \leq \frac{4}{\delta^{2}}\left(1-\left(1-\frac{(1+\delta) \gamma}{n}\right)^{2 n+2}\right)\|p\|_{A^{2}}^{2} \leq \frac{A}{2}\|p\|_{A^{2}}^{2}
\end{aligned}
$$

Consequently,

$$
\begin{aligned}
\sum_{\lambda \in \Lambda_{n}} \frac{|p(\lambda)|^{2}}{k(\lambda, \lambda)} & =\sum_{\lambda \in \Lambda}-\sum_{\lambda \in \Lambda \backslash \Lambda_{n}} \cdots \\
& \geq A\|p\|_{A^{2}}^{2}-\sum_{\lambda \in \Lambda \backslash \Lambda_{n}} \frac{|p(\lambda)|^{2}}{k(\lambda, \lambda)} \geq(A-A / 2)\|p\|_{A^{2}}^{2}
\end{aligned}
$$

Since always $k_{n}(\lambda, \lambda) \leq k(\lambda, \lambda)$, we finish the estimate by

$$
\sum_{\lambda \in \Lambda_{n}} \frac{|p(\lambda)|^{2}}{k_{n}(\lambda, \lambda)} \geq \sum_{\lambda \in \Lambda_{n}} \frac{|p(\lambda)|^{2}}{k(\lambda, \lambda)} \geq \frac{A}{2}\|p\|_{A^{2}}^{2} \quad \text { for all } p \in \mathcal{P}_{n}
$$

As usual, the upper bound is easy. Since by (11) $k_{n}(\lambda, \lambda) \asymp k(\lambda, \lambda)$ for $\lambda \in B_{1-\gamma / n}$, it follows that

$$
\sum_{\lambda \in \Lambda_{n}} \frac{|p(\lambda)|^{2}}{k_{n}(\lambda, \lambda)} \lesssim \gamma \sum_{\lambda \in \Lambda_{n}} \frac{|p(\lambda)|^{2}}{k(\lambda, \lambda)} \leq \sum_{\lambda \in \Lambda} \frac{|p(\lambda)|^{2}}{k(\lambda, \lambda)} \leq B\|p\|_{A^{2}}^{2}
$$

since $\Lambda$ is sampling for $A^{2}(\mathbb{D})$.

Since sampling sets for $A^{2}(\mathbb{D})$ are completely characterized by means of the SeipKorenblum density [24], Theorem 2.11 provides a wealth of examples of Marcinkiewicz-Zygmund families. However, the parameter $\gamma$ in the statement depends on the lower sampling constant $A$ and the separation $\delta$ of $\Lambda$. 


\subsection{From Marcinkiewicz-Zygmund Inequalities to Sampling}

Choose either the Euclidean or the pseudohyperbolic metric on $\mathbb{D}$ and let $d(E, F)$ be the corresponding Hausdorff distance between two closed sets $E, F \subseteq \mathbb{D}$. We say that a sequence of sets $\Lambda_{n} \subseteq \mathbb{D}$ converges weakly to $\Lambda \subseteq \mathbb{D}$, if for all compact disks $B \subseteq \mathbb{D}$

$$
\lim _{n \rightarrow \infty} d\left(\left(\Lambda_{n} \cap B\right) \cup \partial B,(\Lambda \cap B) \cup \partial B\right)=0 .
$$

See $[4,11,13]$ for equivalent definitions. The main consequence of weak convergence is the convergence of sampling sums. If all $\Lambda_{n}$ are uniformly separated with fixed separation $\delta$, then

$$
\sum_{\lambda \in \Lambda_{n} \cap B} \frac{|f(\lambda)|^{2}}{k(\lambda, \lambda)} \rightarrow \sum_{\lambda \in \Lambda \cap B} \frac{|f(\lambda)|^{2}}{k(\lambda, \lambda)}
$$

for all $f \in A^{2}(\mathbb{D})$. More generally, if each $\Lambda_{n}$ is a finite union of $K$ uniformly separated sets with separation constant $\delta$ independent of $n$, then we need to include multiplicities $m(\lambda) \in\{1, \ldots, K\}$. We then obtain

$$
\sum_{\lambda \in \Lambda_{n} \cap B} \frac{|f(\lambda)|^{2}}{k(\lambda, \lambda)} \rightarrow \sum_{\lambda \in \Lambda \cap B} \frac{|f(\lambda)|^{2}}{k(\lambda, \lambda)} m(\lambda) .
$$

Since the multiplicities $m(\lambda)$ are bounded, they only affect the constants, but do not change the arguments.

Theorem 2.12 Assume that $\left(\Lambda_{n}\right)$ is a Marcinkiewicz-Zygmund family for the polynomials $\mathcal{P}_{n}$ in $A^{2}(\mathbb{D})$. Let $\Lambda$ be a weak limit of $\left(\Lambda_{n}\right)$ or of some subsequence $\left(\Lambda_{n_{k}}\right)$. Then $\Lambda$ is a sampling set for $A^{2}(\mathbb{D})$.

Proof Step 1. We assume that $\Lambda_{n}$ is a Marcinkiewicz Zygmund family and therefore there are $A, B>0$ such that $A\|p\|_{A^{2}} \leq \sum_{\lambda \in \Lambda_{n}} \frac{|p(\lambda)|^{2}}{k_{n}(\lambda, \lambda)} \leq B\|p\|_{A^{2}}^{2}$ for all polynomials $p \in \mathcal{P}_{n}$. The upper inequality implies that there are $C>0$ and $\gamma>0$ such that $\#\left(\Lambda_{n} \cap B(w, 0.5(1-|w|))\right) \leq C$ for all $w \in B_{1-\gamma / n}$ and all $n$ (Theorem 2.9). In addition, each set $\Lambda_{n} \cap B_{1-\gamma / n}$ is a finite union of $K$ uniformly separated sequences with separation $\delta$ independent of $n$.

Since $\Lambda_{n}$ converges to $\Lambda$ weakly, $\Lambda$ satisfies the same inequality

$$
\#(\Lambda \cap \bar{B}(w, 0.5(1-|w|))) \leq C, \quad \forall w \in B_{1-\gamma / n}
$$

and thus $\Lambda$ is also a union of $K$ uniformly discrete sequences with separation $\delta$, see, e.g., [4], Sect. 2.1, Lemma 16.

Note that the geometric conditions furnish constants $c_{\gamma}$ for the comparison of the kernels $k$ and $k_{n}$ on $B_{1-\gamma / n}$, the separation constant $\delta$, and the multiplicity $K$. In 
the next step we will choose a suitable radius $r$ that solely depends on these given constants.

Step 2 We prove the desired sampling inequality in $A^{2}(\mathbb{D})$ for all polynomials and then use a density argument.

Fix a polynomial $p$ of degree $N$, say. We know that there is an $r=r(N)<1$ such that $\int_{|z|>r-\delta(1-r)}|p|^{2} \leq \frac{c_{\gamma} \delta^{2} A}{8 K}\|p\|_{A^{2}}^{2}$ because $r-\delta(1-r) \rightarrow 1$ as $r \rightarrow 1$. We take $n \geq N$ big enough such that $r<1-\gamma / n$. In this case we have

$$
\begin{aligned}
A\|p\|_{A^{2}}^{2} \leq \sum_{\lambda \in \Lambda_{n}} \frac{|p(\lambda)|^{2}}{k_{n}(\lambda, \lambda)}= & \sum_{\lambda \in \Lambda_{n},|\lambda|<r} \frac{|p(\lambda)|^{2}}{k_{n}(\lambda, \lambda)} \\
& +\sum_{\lambda \in \Lambda_{n}, r \leq|\lambda|<1-\frac{\gamma}{n}} \frac{|p(\lambda)|^{2}}{k_{n}(\lambda, \lambda)}+\sum_{\lambda \in \Lambda_{n},|\lambda| \geq 1-\frac{\gamma}{n}} \frac{|p(\lambda)|^{2}}{k_{n}(\lambda, \lambda)} \\
= & I_{n}+I I_{n}+I I I_{n} .
\end{aligned}
$$

According to Lemma 2.2 we may replace $k_{n}(\lambda, \lambda)$ by $k(\lambda, \lambda)$ in the first two terms and by $n^{2}$ in the third term. Thus with a constant depending on $\gamma$, we obtain

$$
A\|p\|_{A^{2}}^{2} \leq c_{\gamma}^{-1}\left(\sum_{\lambda \in \Lambda_{n} \cap B_{r}} \frac{|p(\lambda)|^{2}}{k(\lambda, \lambda)}+\sum_{\lambda \in \Lambda_{n}, r \leq|\lambda|<1-\frac{\gamma}{n}} \frac{|p(\lambda)|^{2}}{k(\lambda, \lambda)}+\sum_{\lambda \in \Lambda_{n} \cap C_{\gamma / n}} \frac{|p(\lambda)|^{2}}{n^{2}}\right) .
$$

In the sum $I_{n}$ all points $\lambda$ lie in the compact set $\overline{B(0, r)}$, and by weak convergence including multiplicities $m(\lambda) \in\{1, \ldots, K\}$, we obtain

$$
\begin{aligned}
\lim _{n \rightarrow \infty} I_{n} & \leq c_{\gamma}^{-1} \lim _{n \rightarrow \infty} \sum_{\lambda \in \Lambda_{n} \cap B_{r}} \frac{|p(\lambda)|^{2}}{k(\lambda, \lambda)} \\
& =c_{\gamma}^{-1} \sum_{\lambda \in \Lambda \cap \overline{B_{r}}} \frac{|p(\lambda)|^{2}}{k(\lambda, \lambda)} m(\lambda) \leq c_{\gamma}^{-1} K \sum_{\lambda \in \Lambda \cap \overline{B_{r}}} \frac{|p(\lambda)|^{2}}{k(\lambda, \lambda)} .
\end{aligned}
$$

To treat $I I_{n}$, we use the assertion of Step 1 that every $\Lambda_{n} \cap B_{1-\gamma / n}$ is a finite union of at most $K$ uniformly separated sequences with separation $\delta$. Lemma 2.6, (18) and the choice of $r$ yield

$$
I I_{n} \leq c_{\gamma}^{-1} \sum_{\lambda \in \Lambda_{n}, r \leq|\lambda|<1-\gamma / n} \frac{|p(\lambda)|^{2}}{k(\lambda, \lambda)} \leq \frac{4 K}{\pi \delta^{2} c_{\gamma}} \int_{|z|>r-\delta(1-r)}|p(z)|^{2} \leq \frac{A}{2}\|p\|_{A^{2}}
$$

Finally, the last term $I I I_{n}$ is negligible when $n \rightarrow \infty$ because

$$
I I I_{n} \leq c_{\gamma}^{-1} \sum_{\lambda \in \Lambda_{n} \cap C_{\gamma / n}} \frac{|p(\lambda)|^{2}}{n^{2}} \leq\|p\|_{\infty}^{2} \frac{1}{n^{2}} \#\left(\Lambda_{n} \cap C_{\gamma / n}\right)
$$


By Lemma 2.7(i) $I I I_{n}$ tends to 0 , as $n \rightarrow \infty$.

Finally we take the limit in (26) and obtain inequality

$$
A\|p\|_{A^{2}}^{2} \leq c_{\gamma}^{-1} K \sum_{\lambda \in \Lambda \cap \overline{B(0, r)}} \frac{|p(\lambda)|^{2}}{k(\lambda, \lambda)}+\frac{A}{2}\|p\|_{A^{2}}^{2}
$$

This implies the lower sampling inequality valid for all polynomials.

Since the polynomials are dense in $A^{2}(\mathbb{D}),(27)$ can be extended to all of $A^{2}(\mathbb{D}){ }^{1}$

Step 3 As always, the upper bound is much easier to prove: Let $p \in \mathcal{P}_{N}$. Then by Lemma 2.6

$$
\sum_{\lambda \in \Lambda \cap C_{\gamma / N}} \frac{|p(\lambda)|^{2}}{k(\lambda, \lambda)} \leq C \int_{C_{\gamma^{\prime} / N}}|p(w)|^{2} \mathrm{~d} w \leq C\|p\|_{A^{2}}^{2}
$$

On the disk $B_{1-\gamma / N}$ we use the weak convergence and deduce that

$$
\sum_{\lambda \in \Lambda \cap B_{1-\gamma / N}} \frac{|p(\lambda)|^{2}}{k(\lambda, \lambda)} m(\lambda)=\lim _{n \rightarrow \infty} \sum_{\lambda \in \Lambda_{n} \cap \bar{B}_{1-\gamma / N}} \frac{|p(\lambda)|^{2}}{k_{n}(\lambda, \lambda)} \leq B\|p\|_{A^{2}}^{2}
$$

because $\Lambda_{n}$ is a Marcinkiewicz-Zygmund family for polynomials and $p \in \mathcal{P}_{N} \subseteq \mathcal{P}_{n}$. The sums of both terms yield the upper sampling inequality for $\Lambda$ for all polynomials, which extends to $A^{2}(\mathbb{D})$ by density.

The upper bound can also be derived from the geometric description of Theorem 2.9.

With a bit more effort one can prove an $A^{p}$-version of Theorems 2.11 and 2.12. The proof requires several modifications of interest. For instance, to obtain (7) for the $A^{p}$-norm, one needs an argument similar to [22, Lemma 7]. The $A^{p}$-norm of the normalized reproducing kernel in Proposition 2.7 requires the boundedness of the Bergman projection on $L^{p}$.

\section{Hardy Space}

\subsection{Basic Facts}

The Hardy space $H^{2}=H^{2}(\mathbb{D})$ consists of all analytic functions in $\mathbb{D}$ whose boundary values on $\partial \mathbb{D}=\mathbb{T}$ are in $L^{2}(\mathbb{T})$ with finite norm

$$
\|f\|_{H^{2}}=\left(\int_{0}^{1}\left|f\left(e^{2 \pi i t}\right)\right|^{2} d t\right)^{1 / 2} .
$$

\footnotetext{
1 Note that $\sum_{\lambda \in \Lambda} \frac{|p(\lambda)|^{2}}{k(\lambda, \lambda)}=\sum_{\lambda \in \Lambda}\left|\left\langle p, \kappa_{\lambda}\right\rangle\right|^{2}$, where $\kappa_{\lambda}(z)=k(z, \lambda) k(\lambda, \lambda)^{-1 / 2}$ is the normalized reproducing kernel. Thus the left-hand side in (27) is just the frame operator associated to the set $\left\{\kappa_{\lambda}\right\}$. For boundedness and invertibility it therefore suffices to check on a dense subset.
} 
The monomials $z \rightarrow z^{k}$ form an orthonormal basis, and the norm of $f(z)=$ $\sum_{k=0}^{\infty} a_{k} z^{k}$ is

$$
\|f\|_{H^{2}}^{2}=\sum_{k=0}^{\infty}\left|a_{k}\right|^{2} .
$$

For $p(z)=\sum_{k=0}^{n} a_{k} z^{k} \in \mathcal{P}_{n}$ and $0<\rho \leq 1$, let $p_{\rho}(z)=p(\rho z)$, then

$$
\left\|p_{\rho}\right\|_{H^{2}}^{2}=\sum_{k=0}^{n}\left|a_{k}\right|^{2} \rho^{2 k} \geq \rho^{2 n}\|p\|_{H^{2}}^{2},
$$

and clearly $\left\|p_{\rho}\right\|_{H^{2}} \leq\|p\|_{H^{2}}$. If $p \in \mathcal{P}_{n}, \rho=1-\frac{\gamma}{n}$, and $n>2 \gamma$, then by (8)

$$
\|p\|_{H^{2}}^{2} \geq\left\|p_{\rho}\right\|_{H^{2}}^{2} \geq\left(1-\frac{\gamma}{n}\right)^{2 n}\|p\|_{H^{2}}^{2} \geq e^{-4 \gamma}\|p\|_{H^{2}}^{2} .
$$

The reproducing kernel of $\mathcal{P}_{n}$ in $H^{2}$ is given by

$$
k_{n}(z, w)=\sum_{k=0}^{n}(z \bar{w})^{n}=\frac{1-(z \bar{w})^{n+1}}{1-z \bar{w}}
$$

As $n \rightarrow \infty$, the kernel tends to the reproducing kernel of $H^{2}$,

$$
k(z, w)=\frac{1}{1-z \bar{w}}
$$

for $z, w \in \mathbb{D}$. For Marcinkiewicz-Zygmund families for polynomials in Hardy space it will be necessary to also consider sampling points outside the unit disk as in [22]. With this caveat in mind, we define the appropriate annuli as

$$
A_{\gamma / n}=\left\{z \in \mathbb{C}: 1-\frac{\gamma}{n} \leq|z| \leq\left(1-\frac{\gamma}{n}\right)^{-1}\right\}
$$

We now compare the kernels for $\mathcal{P}_{n}$ and $H^{2}$.

Lemma 3.1 Let $k_{n}(z, w)$ be the reproducing kernel of $\mathcal{P}_{n}$ in $H^{2}$ and let $\gamma>0$ be arbitrary.

If $z \in A_{\gamma / n}$, i.e., $1-\frac{\gamma}{n} \leq|z| \leq\left(1-\frac{\gamma}{n}\right)^{-1}$, and $n>2 \gamma$, then

$$
\frac{1-e^{-2 \gamma}}{2 \gamma} n \leq k_{n}(z, z) \leq \frac{e^{4 \gamma}}{\gamma} n .
$$

Consequently, if $\Lambda_{n} \subseteq A_{\gamma / n}$, then, for all polynomials $p \in \mathcal{P}_{n}$,

$$
\frac{1}{n} \sum_{\lambda \in \Lambda_{n}}|p(\lambda)|^{2} \asymp \sum_{\lambda \in \Lambda_{n}} \frac{|p(\lambda)|^{2}}{k_{n}(\lambda, \lambda)} .
$$


On the annulus $A_{\gamma / n}$ we may therefore always work with the weight $1 / n$ for polynomials of degree $n$.

Proof Writing $r=|z|$, the kernel $k_{n}(z, z)=\frac{1-r^{2 n+2}}{1-r^{2}}$ is increasing in $r$, so that for $z \in A_{\gamma / n}$ we have

$$
\frac{1-\left(1-\frac{\gamma}{n}\right)^{2 n+2}}{1-\left(1-\frac{\gamma}{n}\right)^{2}} \leq k_{n}(z, z) \leq \frac{\left(1-\frac{\gamma}{n}\right)^{-2 n-2}-1}{\left(1-\frac{\gamma}{n}\right)^{-2}-1}=\frac{\left(1-\frac{\gamma}{n}\right)^{-2 n}-\left(1-\frac{\gamma}{n}\right)^{2}}{1-\left(1-\frac{\gamma}{n}\right)^{2}} .
$$

For $n \geq 2 \gamma$, the denominator $1-\left(1-\frac{\gamma}{n}\right)^{2}=\frac{\gamma}{n}+\frac{\gamma}{n}\left(1-\frac{\gamma}{n}\right)$ is between $\gamma / n$ and $2 \gamma / n$, for the numerator we use (8) and obtain $\left(1-\frac{\gamma}{n}\right)^{-2 n}-\left(1-\frac{\gamma}{n}\right)^{2} \leq e^{4 \gamma}$ and $1-\left(1-\frac{\gamma}{n}\right)^{2 n+2} \geq 1-\left(1-\frac{\gamma}{n}\right)^{2 n} \geq 1-e^{-2 \gamma}$. Thus both ratios are of order $n$ with the constants $\left(1-e^{-2 \gamma}\right) /(2 \gamma)$ and $e^{4 \gamma} / \gamma$.

\subsection{From Marcinkiewicz-Zygmund Families on $\mathbb{T}$ to Marcinkiewicz-Zygmund Families for $H^{2}(\mathbb{D})$}

In contrast to the situation for the Bergman space, there are no sampling sequences for the Hardy space $H^{2}(\mathbb{D})$ by the results of Thomas [27]. Duren and Schuster [4, p. 154] give the following simple argument: a sampling set $\Lambda$ for $H^{2}$ must be a Blaschke sequence. However, every Blaschke sequence is a zero set in $H^{2}$, which contradicts the sampling inequality. Therefore there can be no analogue of Theorem 2.11.

Nevertheless, we prove that Hardy space admits Marcinkiewicz-Zygmund families for polynomials. The idea is to associate a Marcinkiewicz-Zygmund family for polynomials in $H^{2}(\mathbb{D})$ to every Marcinkiewicz-Zygmund family for polynomials on the torus. As these are well understood [8,22], we obtain a general class of Marcinkiewicz-Zygmund families in $H^{2}(\mathbb{D})$.

We use the following notation: for $\lambda \in \mathbb{C} \backslash\{0\}$ let $\tilde{\lambda}=\frac{\lambda}{|\lambda|}$ be the projection from the complex plane $\mathbb{C} \backslash\{0\}$ onto the torus $\partial \mathbb{D}=\mathbb{T}$.

Theorem 3.2 Assume that the family $\left(\Lambda_{n}\right) \subseteq \mathbb{C}$ has the following properties:

(i) there exists $\gamma>0$, such that $\Lambda_{n} \subseteq A_{\gamma / n}$ for all $n \geq \gamma$.

(ii) The projected family $\left(\tilde{\Lambda}_{n}\right) \subseteq \mathbb{T}$ is a Marcinkiewicz-Zygmund family for the polynomials $\mathcal{P}_{n} \subseteq L^{2}(\mathbb{T})$.

(iii) The projection $\Lambda_{n} \rightarrow \widetilde{\Lambda_{n}}$ from $A_{\gamma / n}$ to $\mathbb{T}$ is one-to-one for all $n$.

Then $\left(\Lambda_{n}\right)$ is a Marcinkiewicz-Zygmund family for the polynomials $\mathcal{P}_{n}$ in $H^{2}(\mathbb{D})$.

The statement in the introduction is just a reformulation of Theorem 3.2 without additional notation.

The proof is inspired by a sampling theorem of Duffin and Schaeffer for bandlimited functions from samples in the complex plane (rather than from samples on the real axis). See [5] and [25,28]. In analogy to the theory of bandlimited functions, one can view Theorem 3.2 also as a perturbation result for Marcinkiewicz-Zygmund families in $L^{2}(\mathbb{T})$, where the points in $\mathbb{T}$ are perturbed in a complex neighborhood of $\mathbb{T}$.

We wrap the main part of the proof into a technical lemma. 
Lemma 3.3 Let $\gamma>0$ and $n>4 \gamma$. Assume that $\Lambda_{n}$ is a finite set contained in $A_{\gamma / n}$ so that the projection $\Lambda_{n} \rightarrow \widetilde{\Lambda_{n}}$ is one-to-one. Then there exists a set $\Lambda_{n}^{(1)}$ with the following properties:

(i) $\Lambda_{n}^{(1)}$ is contained in the smaller annulus $A_{\frac{3 \gamma}{4 n}}$ and $\# \Lambda_{n}^{(1)}=\# \Lambda_{n}$.

(ii) For every $\lambda \in \Lambda_{n}$ there is a $\mu \in \Lambda_{n}^{(1)}$, such that $\tilde{\lambda}=\tilde{\mu}$, and

(iii) For every $p \in \mathcal{P}_{n}$ there exists $p_{1} \in \mathcal{P}_{n}$ satisfying

$$
\left\|p_{1}\right\|_{H^{2}}^{2} \geq e^{-2 \gamma / 3}\|p\|_{H^{2}}^{2}
$$

and

$$
\frac{1}{n} \sum_{\lambda \in \Lambda_{n}}|p(\lambda)|^{2} \geq \frac{1}{n} \sum_{\lambda \in \Lambda_{n}^{(1)}}\left|p_{1}(\lambda)\right|^{2}
$$

As we will apply the lemma to a Marcinkiewicz-Zygmund family, it is important the constants and the construction are independent of the degree $n$.

Proof Step 1. Construction of $\Lambda_{n}^{(1)}$. If $\lambda \in \Lambda_{n}$ and $|\lambda| \leq 1$, set $\mu=\left(1+\frac{\gamma}{3 n}\right) \lambda$. If $\lambda \in \Lambda_{n}$ and $|\lambda|>1$, set $\mu=\left(1+\frac{\gamma}{3 n}\right) / \bar{\lambda}$. By construction, $\tilde{\mu}=\tilde{\lambda}$. Since $\Lambda_{n} \rightarrow \widetilde{\Lambda_{n}}$ is one-to-one by assumption, $\Lambda_{n}^{(1)}$ has the same cardinality as $\Lambda_{n}$.

[To appreciate this assumption, consider the case when both $\lambda$ and $1 / \bar{\lambda}$ are in $\Lambda_{n}$. They both would be mapped to the same point $\mu$.] If $1-\frac{\gamma}{n} \leq|\lambda| \leq 1$ and $n>4 \gamma$, then $\mu=\left(1+\frac{\gamma}{3 n}\right) \lambda$ satisfies the inequalities

$$
1-\frac{3 \gamma}{4 n} \leq\left(1+\frac{\gamma}{3 n}\right)\left(1-\frac{\gamma}{n}\right) \leq|\mu| \leq\left(1+\frac{\gamma}{3 n}\right) \leq\left(1-\frac{3 \gamma}{4 n}\right)^{-1} .
$$

If $1<|\lambda| \leq\left(1-\frac{\gamma}{n}\right)^{-1}$, then $\mu=\left(1+\frac{\gamma}{3 n}\right) / \bar{\lambda}$ satisfies the same inequalities. It follows that $\Lambda_{n}^{(1)}$ is contained in the smaller annulus $A_{3 \gamma /(4 n)}$.

Step 2. Construction of $p_{1}$. Given $p \in \mathcal{P}_{n}$ with zeros $z_{j}$ and factorization $p(z)=$ $z^{\ell} \prod\left(z-z_{j}\right)$, we obtain $p_{1}$ by reflecting all its zeros into $\mathbb{D}$ and an appropriate scaling. We multiply $p$ by several Blaschke factors and set

$$
\widetilde{p_{1}}(z)=z^{l} \prod_{\left|z_{j}\right| \leq 1}\left(z-z_{j}\right) \prod_{\left|z_{j}\right|>1}\left(1-\overline{z_{j}} z\right)=p(z) \prod_{\left|z_{j}\right|>1} \frac{1-\overline{z_{j}} z}{z-z_{j}} .
$$

By construction, all zeros of $\tilde{p}_{1}$ are now in the unit disk $\overline{\mathbb{D}}$. In engineering terminology, $\widetilde{p_{1}}$ is the minimum phase filter associated to $p$. Furthermore, $\widetilde{p_{1}}$ has the following properties:

(i) By (37) and the property of Blaschke factors we have

$$
\left|\widetilde{p_{1}}(z)\right|=|p(z)| \quad \text { for } z \in \mathbb{T},
$$

and thus $\left\|\tilde{p}_{1}\right\|_{H^{2}}=\|p\|_{H^{2}}$. 
(ii) For $z \in \mathbb{D}$ we have

$$
\left|\widetilde{p_{1}}(z)\right| \leq \min (|p(z)|,|p(1 / \bar{z})|)
$$

To see this, observe that for $\left|z_{j}\right|>1$ each Blaschke factor in (37) satisfies

$$
\left|\frac{1-\bar{z}_{j} z}{z-z_{j}}\right|=\left|\frac{\bar{z}_{j}^{-1}-z}{z_{j}^{-1} z-1}\right| \leq 1
$$

thus $\left|\widetilde{p_{1}}(z)\right| \leq|p(z)|$ for $z \in \mathbb{D}$. Using the first factorization of $\widetilde{p_{1}}$ in (37) and $p(1 / \bar{z})=\bar{z}^{-l} \prod_{\left|z_{j}\right| \leq 1}\left(1 / \bar{z}-z_{j}\right) \prod_{\left|z_{j}\right|>1}\left(1 / \bar{z}-z_{j}\right)$, the second inequality in (38) follows from

$$
\frac{\left|\tilde{p}_{1}(z)\right|}{\left|p\left(\frac{1}{\bar{z}}\right)\right|}=|z|^{2 l} \prod_{\left|z_{j}\right| \leq 1} \frac{\left|z-z_{j}\right|}{\left|\bar{z}^{-1}\left(1-\bar{z} z_{j}\right)\right|} \prod_{\left|z_{j}\right|>1} \frac{\left|1-\overline{z_{j}} z\right|}{\left|\bar{z}^{-1}\left(1-\bar{z} z_{j}\right)\right|} \leq 1 .
$$

Finally we set

$$
p_{1}(z)=\tilde{p}_{1}\left(\left(1+\frac{\gamma}{3 n}\right)^{-1} z\right) \in \mathcal{P}_{n}
$$

Then by (30)

$$
\left\|p_{1}\right\|_{H^{2}}^{2} \geq\left(1+\frac{\gamma}{3 n}\right)^{-2 n}\left\|\widetilde{p}_{1}\right\|_{H^{2}}^{2} \geq e^{-2 \gamma / 3}\|p\|_{H^{2}}^{2}
$$

and obviously $\left\|p_{1}\right\|_{H^{2}}^{2} \leq\left\|\tilde{p}_{1}\right\|_{H^{2}}^{2}=\|p\|_{H^{2}}^{2}$.

Step 3. Sampling on $\Lambda_{n}^{(1)}$. If $\mu=\left(1+\frac{\gamma}{3 n}\right) \lambda \in \Lambda_{n}^{(1)} \subseteq \mathbb{D}$, then by (38)

$$
\left|p_{1}(\mu)\right|=\left|\widetilde{p_{1}}(\lambda)\right| \leq|p(\lambda)|
$$

if $\mu=\left(1+\frac{\gamma}{3 n}\right) / \bar{\lambda} \in \Lambda_{n}^{(1)} \subseteq \mathbb{D}$, then

$$
\left|p_{1}(\mu)\right|=\left|\tilde{p}_{1}(1 / \bar{\lambda})\right| \leq|p(\lambda)| .
$$

In conclusion, we obtain

$$
\frac{1}{n} \sum_{\mu \in \Lambda_{n}^{(1)}}\left|p_{1}(\mu)\right|^{2} \leq \frac{1}{n} \sum_{\lambda \in \Lambda_{n}}|p(\lambda)|^{2}
$$

which was to be shown. 
Proof of Theorem 3.2 Applying Lemma 3.3 repeatedly, after $\ell$ steps we construct a set $\Lambda_{n}^{(\ell)} \subseteq \mathbb{D}$ with the following properties:

$$
\begin{aligned}
& \Lambda_{n}^{(\ell)} \subseteq A_{\gamma_{\ell} / n} \quad \text { with } \gamma_{\ell}=\frac{3}{4} \gamma_{\ell-1}, \\
& \Lambda_{n}^{(\ell)}=\widetilde{\Lambda_{n}} .
\end{aligned}
$$

Furthermore, for given $p \in \mathcal{P}_{n}$ we construct a sequence on polynomials $p_{1}, \ldots, p_{\ell}, \ldots$ with decreasing norm $\left\|p_{\ell}\right\|_{H^{2}} \leq \cdots \leq\left\|p_{1}\right\|_{H^{2}} \leq\|p\|_{H^{2}}$, such that

$$
\left\|p_{\ell}\right\|_{H^{2}}^{2} \geq e^{-\frac{2}{3} \gamma_{\ell-1}}\left\|p_{\ell-1}\right\|_{H^{2}}^{2} \geq e^{-\frac{2}{3}\left(\gamma_{\ell-1}+\gamma_{\ell-2}\right)}\left\|p_{\ell-2}\right\|_{H^{2}}^{2} \geq \ldots \geq e^{-\frac{2}{3} \sum_{j=0}^{\ell-1} \gamma_{j}}\|p\|_{H^{2}}^{2} .
$$

Since $\gamma_{j}=3 \gamma_{j-1} / 4$ and $\gamma_{0}=\gamma$, we find $\gamma_{\ell}=\left(\frac{3}{4}\right)^{\ell} \gamma$ and

$$
\frac{2}{3} \sum_{j=0}^{\ell-1} \gamma_{j} \leq \frac{2}{3} \sum_{j=0}^{\infty}(3 / 4)^{j} \gamma \leq \frac{8}{3} \gamma
$$

It follows that always $\left\|p_{\ell}\right\|_{H^{2}}^{2} \geq e^{-\frac{8}{3} \gamma}\|p\|_{H^{2}}^{2}$.

We now let $\ell$ tend to $\infty$. Since the sequence $p_{\ell}$ is bounded in the finite-dimensional space $\mathcal{P}_{n}$, it contains a convergent subsequence such that $\lim _{j \rightarrow \infty} p_{\ell_{j}}=p_{\infty} \in \mathcal{P}_{n}$. Furthermore, by (42) the limiting polynomial $p_{\infty}$ must be non-zero. By (40) every point in $\Lambda_{n}^{(\ell)}$ converges to a point on the torus, precisely to the corresponding point in the projection $\widetilde{\Lambda_{n}}$. Using (42), it follows that

$$
\frac{\frac{1}{n} \sum_{\lambda \in \Lambda_{n}}|p(\lambda)|^{2}}{\|p\|_{H^{2}}^{2}} \geq e^{-8 \gamma / 3} \frac{\frac{1}{n} \sum_{\lambda \in \widetilde{\Lambda}_{n}}\left|p_{\infty}(\lambda)\right|^{2}}{\left\|p_{\infty}\right\|_{H^{2}}^{2}} .
$$

Finally, we recall the assumption that the projected family $\widetilde{\Lambda_{n}}$ is a Marcinkiewicz-Zygmund family for the polynomials of degree $n$ in $L^{2}(\mathbb{T})$. Consequently, we obtain that $\frac{1}{n} \sum_{\lambda \in \widetilde{\Lambda_{n}}}\left|p_{\infty}(\lambda)\right|^{2} \geq A\left\|p_{\infty}\right\|_{H^{2}}^{2}$, which implies the corresponding sampling inequality for $p \in \mathcal{P}_{n}$.

The upper bound is proved almost exactly as the corresponding ${ }^{2}$ statement for $\mathcal{P}_{n}$ in $L^{2}(\mathbb{T})$ in Thm. 9 of [22]. Since $\widetilde{\Lambda}_{n}$ is a Marcinkiewicz-Zygmund family for $\mathcal{P}_{n}$ in $L^{2}(\mathbb{T})$ by our assumption, [22, Thm. 9] asserts that for every interval $I \subseteq \mathbb{T}$ of length $1 / n$ we have $\#\left(\widetilde{\Lambda_{n}} \cap I\right) \leq C$. Since $\Lambda_{n} \subseteq A_{\gamma / n}$, this condition implies that $\# \Lambda_{n} \cap B(z, 1 / n) \leq C^{\prime}$ for all $z \in A_{\gamma / n}$. This geometric condition now yields the upper bound $\frac{1}{n} \sum_{\lambda \in \Lambda_{n}}|p(\lambda)|^{2} \lesssim\|p\|_{H^{2}}^{2}$ for all $p \in \mathcal{P}_{n}$ precisely as in [22]. Indeed that proof uses the submean-value property and the extension to $A_{\gamma / n}$.

Theorem 3.2 shows that to every Marcinkiewicz-Zygmund family for polynomials on $\mathbb{T}$ we can associate Marcinkiewicz-Zygmund families in $H^{2}(\mathbb{D})$ by moving points from the boundary $\mathbb{T}=\partial \mathbb{D}$ into a carefully controlled annulus $C_{\gamma / n} \subseteq \mathbb{D}$. The

\footnotetext{
2 Note that [22] uses the weights $m_{n}=\# \Lambda_{n}$ instead of $k_{n}(\lambda, \lambda) \asymp n$. This does not affect the estimates.
} 
following example investigates the role of points in the interior of $\mathbb{D}$ for Marcinkiewicz-Zygmund families.

Example We construct an example of a Marcinkiewicz-Zygmund family $\left(\Lambda_{n}\right)$ for polynomials $\mathcal{P}_{n}$ in $H^{2}(\mathbb{D})$, so that $\left(\Lambda_{n} \cap C_{\gamma / n}\right)$ is not a Marcinkiewicz-Zygmund family in $H^{2}$ and the projection $\left(\widetilde{\Lambda_{n}}\right)$ is not a Marcinkiewicz-Zygmund family for $\mathcal{P}_{n}$ in $L^{2}(\mathbb{T})$. Let $\gamma>0, \alpha_{n}>0$, and

$$
\Lambda_{n}=\left\{\left(1-\frac{\gamma}{n}\right) e^{2 \pi i k / n}: k=0, \ldots, n-1\right\} \cup\left\{\alpha_{n} e^{2 \pi i / n^{2}}\right\} .
$$

(i) If $\alpha_{n}<1-\frac{\gamma}{n}$, then $\#\left(\Lambda_{n} \cap C_{\gamma / n}\right)=n<\operatorname{dim} \mathcal{P}_{n}$ and thus $\left(\Lambda_{n} \cap C_{\gamma / n}\right)$ cannot be a Marcinkiewicz-Zygmund family for $\mathcal{P}_{n}$ in $H^{2}$.

(ii) The projected family $\widetilde{\Lambda_{n}}=\left\{e^{2 \pi i k / n}: k=0, \ldots, n-1\right\} \cup\left\{e^{2 \pi i / n^{2}}\right\}$ is not a Marcinkiewicz-Zygmund family for $\mathcal{P}_{n}$ in $L^{2}(\mathbb{T})$. We choose $p(z)=z^{n}-1$ with $\|p\|_{H^{2}}^{2}=2$. Then $p\left(e^{2 \pi i k / n}\right)=0$ and $\left|p\left(e^{2 \pi i / n^{2}}\right)\right|=\left|e^{2 \pi i / n}-1\right| \lesssim 1 / n$, so that

$$
\frac{1}{n+1} \sum_{\lambda \in \widetilde{\Lambda_{n}}}|p(\lambda)|^{2} \lesssim \frac{1}{n^{3}}
$$

violating the sampling inequality for large $n$.

(iii) However, $\left(\Lambda_{n}\right)$ is a Marcinkiewicz-Zygmund family for $\mathcal{P}_{n}$ in $H^{2}(\mathbb{D})$. To see this, we consider the modified set $\Lambda_{n}^{\prime}=\left\{\left(1-\frac{\gamma}{n}\right) e^{2 \pi i k / n}: k=0, \ldots, n-1\right\} \cup\{0\}$ and then use a perturbation argument.

We write $p \in \mathcal{P}_{n}$ as $p(z)=p(0)+z \tilde{p}(z)$ for a unique $\tilde{p} \in \mathcal{P}_{n-1}$. Then $\|p\|_{H^{2}}^{2}=$ $|p(0)|^{2}+\|\tilde{p}\|_{H^{2}}^{2}$. Let $q(z)=\tilde{p}\left(\left(1-\frac{\gamma}{n}\right) z\right)$, then by (30) $\|q\|_{H^{2}}^{2} \geq e^{-4 \gamma}\|\widetilde{p}\|_{H^{2}}^{2}$. Calculating the norm of $q$ by sampling, we obtain

$$
\begin{aligned}
\|\tilde{p}\|^{2} \asymp\|q\|^{2} & =\frac{1}{n} \sum_{j=0}^{n-1}\left|q\left(e^{2 \pi i j / n}\right)\right|^{2}=\frac{1}{n} \sum_{j=0}^{n-1}\left|\tilde{p}\left(\left(1-\frac{\gamma}{n}\right) e^{2 \pi i j / n}\right)\right|^{2} \\
& =\frac{1}{n\left(1-\frac{\gamma}{n}\right)^{2}} \sum_{j=0}^{n-1}\left|\left(1-\frac{\gamma}{n}\right) e^{2 \pi i j / n} \widetilde{p}\left(\left(1-\frac{\gamma}{n}\right) e^{2 \pi i j / n}\right)\right|^{2} .
\end{aligned}
$$

If $n \geq 2 \gamma$ we have

$$
\|q\|^{2} \leq \frac{8}{n} \sum_{j=0}^{n-1}\left(\left|p\left(\left(1-\frac{\gamma}{n}\right) e^{2 \pi i j / n}\right)\right|^{2}+|p(0)|^{2}\right) .
$$

Since by (34) $k_{n}(\lambda, \lambda) \asymp n$ for $\lambda=\left(1-\frac{\gamma}{n}\right) e^{2 \pi i j / n}$, and $k_{n}(0,0)=1$, the above inequality states that

$$
\|q\|^{2} \lesssim \sum_{\lambda \in \Lambda_{n}^{\prime}} \frac{|p(\lambda)|^{2}}{k_{n}(\lambda, \lambda)}
$$


and finally

$$
\|p\|^{2} \leq\|q\|^{2}+|p(0)|^{2} \lesssim \sum_{\lambda \in \Lambda_{n}^{\prime}} \frac{|p(\lambda)|^{2}}{k_{n}(\lambda, \lambda)}
$$

Thus $\left(\Lambda_{n}^{\prime}\right)$ is a Marcinkiewicz-Zygmund family for $\mathcal{P}_{n}$ in $H^{2}(\mathbb{D})$. The small perturbation $0 \rightarrow \frac{1}{n^{2}} e^{2 \pi i / n^{2}}$ is of order $1 / n^{2}$ and thus preserves the sampling inequality.

As already mentioned, the standard definition of sampling sequences is vacuous in the Hardy space. Thus Thomas in [27] proposed an alternative definition of sampling in terms of the non-tangential maximal function $M_{\Lambda}$.

$$
M_{\Lambda}(f)\left(e^{i \theta}\right):=\sup _{\Gamma\left(e^{i \theta}\right) \cap \Lambda}|f|
$$

where $\Gamma\left(e^{i \theta}\right)=\left\{z \in \mathbb{D}: \frac{\left|z-e^{i \theta}\right|}{1-|z|}<1+\alpha\right\}$ is a non-tangential Stolz angle at the point $e^{i \theta}$. A set $\Lambda$ is called PT-sampling ${ }^{3}$ in $H^{2}(\mathbb{D})$ if $\left\|M_{\Lambda}(f)\right\|_{L^{2}} \gtrsim\|f\|_{2}$ for all $f \in H^{2}(\mathbb{D})$.

Thomas proves that a set $\Lambda$ is sampling for $H^{2}$ if and only if it is norming for $H^{\infty}$, this condition was geometrically described by Brown et al. [2] by the property that the non-tangential limit set of $\Lambda$ must be of full measure in $\mathbb{T}$. This alternative notion of sampling was inspired by a corresponding alternative definition of interpolating sequences in the Hardy space by Bruna, Nicolau and Øyma [3].

The relation between Marcinkiewicz-Zygmund families and PT-sampling sets for $H^{2}$ is not clear. We only mention that there is no analog of Theorem 2.12 for PTsampling: Consider the Marcinkiewicz-Zygmund family $\Lambda_{n}$ in the example (43). Its weak limit in $\mathbb{D}$ is just $\{0\}$, which is obviously not PT-sampling. Its weak limit in $\mathbb{C}$ is $\{0\} \cup \partial \mathbb{D}$, and this not even covered by the definition of PT-sampling. We have not pursued this aspect further.

Funding Open Access funding provided by University of Vienna.

Open Access This article is licensed under a Creative Commons Attribution 4.0 International License, which permits use, sharing, adaptation, distribution and reproduction in any medium or format, as long as you give appropriate credit to the original author(s) and the source, provide a link to the Creative Commons licence, and indicate if changes were made. The images or other third party material in this article are included in the article's Creative Commons licence, unless indicated otherwise in a credit line to the material. If material is not included in the article's Creative Commons licence and your intended use is not permitted by statutory regulation or exceeds the permitted use, you will need to obtain permission directly from the copyright holder. To view a copy of this licence, visit http://creativecommons.org/licenses/by/4.0/.

\section{References}

1. Bergman, S.: The Kernel Function and Conformal Mapping. Mathematical Surveys and Mongraphs. AMS, New York (1950)

\footnotetext{
3 For Pascal Thomas.
} 
2. Brown, L., Shields, A., Zeller, K.: On absolutely convergent exponential sums. Trans. Am. Math. Soc. 96, 162-183 (1960)

3. Bruna, J., Nicolau, A., Øyma, K.: A note on interpolation in the Hardy spaces of the unit disc. Proc. Am. Math. Soc. 124(4), 1197-1204 (1996)

4. Duren, P., Schuster, A.: Bergman Spaces. Mathematical Surveys and Monographs. American Mathematical Society, Providence (2004)

5. Duffin, R.J., Schaeffer, A.C.: A class of nonharmonic Fourier series. Trans. Am. Math. Soc. 72, 341-366 (1952)

6. Erdélyi, T.: Notes on inequalities with doubling weights. J. Approx. Theory 100(1), 60-72 (1999)

7. Filbir, F., Mhaskar, H.N.: Marcinkiewicz-Zygmund measures on manifolds. J. Complex. 27(6), 568596 (2011)

8. Gröchenig, K.: Irregular sampling, Toeplitz matrices, and the approximation of entire functions of exponential type. Math. Comput. 68(226), 749-765 (1999)

9. Gröchenig, K.: Non-Uniform Sampling in Higher Dimensions: From Trigonometric Polynomials to Band-Limited Functions. In: Benedetto, J.J., Ferreira, P. (eds.) Non-Uniform Sampling in Higher Dimensions: From Trigonometric Polynomials to Band-Limited Functions, pp. 155-171. Birkhäuser, Boston (2001)

10. Gröchenig, K., Ortega-Cerdà, J.: Marcinkiewicz-Zygmund families in Fock space and Hermite polynomials (in preparation)

11. Gröchenig, K., Ortega-Cerdà, J., Romero, J.L.: Deformation of Gabor systems. Adv. Math. 277, 388425 (2015)

12. Hastings, W.W.: A Carleson measure theorem for Bergman spaces. Proc. Am. Math. Soc. 52, 237-241 (1975)

13. Hedenmalm, H., Korenblum, B., Zhu, K.: Theory of Bergman Spaces. Graduate Texts in Mathematics, vol. 199. Springer, New York (2000)

14. Lubinsky, D.S.: Marcinkiewicz-Zygmund inequalities: methods and results. In: Recent progress in inequalities (Niš, 1996), vol. 430 Math. Appl., pp. 213-240. Kluwer Academic Publisher, Dordrecht (1998)

15. Lubinsky, D.S.: On converse Marcinkiewicz-Zygmund inequalities in $L_{p}, p>1$. Constr. Approx. 15(4), 577-610 (1999)

16. Marzo, J.: Marcinkiewicz-Zygmund inequalities and interpolation by spherical harmonics. J. Funct. Anal. 250(2), 559-587 (2007)

17. Marzo, J., Ortega-Cerdà, J.: Equidistribution of Fekete points on the sphere. Constr. Approx. 32(3), 513-521 (2010)

18. Mastroianni, G., Totik, V.: Weighted polynomial inequalities with doubling and $A_{\infty}$ weights. Constr. Approx. 16(1), 37-71 (2000)

19. Mhaskar, H.N., Narcowich, F.J., Ward, J.D.: Spherical Marcinkiewicz-Zygmund inequalities and positive quadrature. Math. Comput. 70(235), 1113-1130 (2001)

20. Nevai, P.: Géza Freud, orthogonal polynomials and Christoffel functions. A case study. J. Approx. Theory 48(1), 3-167 (1986)

21. Ortega-Cerdà, J., Pridhnani, B.: Beurling-Landau's density on compact manifolds. J. Funct. Anal. 263(7), 2102-2140 (2012)

22. Ortega-Cerdà, J., Saludes, J.: Marcinkiewicz-Zygmund inequalities. J. Approx. Theory 145(2), 237252 (2007)

23. Seip, K.: Regular sets of sampling and interpolation for weighted Bergman spaces. Proc. Am. Math. Soc. 117(1), 213-220 (1993)

24. Seip, K.: Beurling type density theorems in the unit disk. Invent. Math. 113(1), 21-39 (1993)

25. Seip, K.: Interpolation and Sampling in Spaces of Analytic Functions. University Lecture Series, vol. 33. American Mathematical Society, Providence (2004)

26. Shapiro, H.S., Shields, A.: On some interpolation problems for analytic functions. Am. J. Math. 83, 513-532 (1961)

27. Thomas, P.J.: Sampling sets for Hardy spaces of the disk. Proc. Am. Math. Soc. 126(10), 2927-2932 (1998)

28. Young, R.M.: An Introduction to Nonharmonic Fourier Series. Academic Press, New York (1980)

Publisher's Note Springer Nature remains neutral with regard to jurisdictional claims in published maps and institutional affiliations. 